\title{
Presenteeism: The Productivity Paradox
}

\author{
Dr. Vijit Chaturvedi ${ }^{*}$, Prof. Sanjeev Bansal ${ }^{2}$ \\ ${ }^{I}$ Professor, Amity Business School, Amity University, Noida, Uttar Pradesh \\ ${ }^{2}$ Dean, Faculty of Management Studies, Amity Business School, Amity University, Noida, Uttar Pradesh
}

\begin{abstract}
As organization are trying to achieve more by less it is evident that the pressure is increasing on the physical and mental health of employees in varied form. Presenteeism refers to reporting at workplace even when ill or even if unwell. This approach is slowly increasing due to the rising reassure of remaining competitive and excelling others in the unending race of competition. This is a more serious issue of concern as the data and conditions of absenteeism can be recorded, measured and diagnosed but presenteeism is comparatively difficult to assess and work upon. In fact presenteeism seems to be affecting the health of the employees much core than absenteeism thus requires a workplace attention in order to preserve the future vitality and organization credibility as a preferred place to work.
\end{abstract}

The present paper emphasizes on various aspects of this concern with respect to the available factors leading to presenteeism, causes and suitable measures to reduce the dysfunctional outcome of this. It also highlights different measures available to measure presenteeism highlighting its striking features from absenteeism. The discussion will aid in bringing forth the relevance of such pertinent issue ensuring long term employee health, organization culture and climate practices intact and ensuring healthy employer employee relation along with employee productivity and efficiency.

Keywords - Self-esteem; Resilience; College Students

\section{Introduction}

Presenteeism relates to severe situation wherein an employee is not well but still keeps coming to organization and this has caused directly and indirectly affects both organisation, employees physical, mental and psychological health and also affects the long term retention and possibly leading to absenteeism also. Thus, the subject is of prime importance not only a concern for organization leadership or organization commitment studies but largely with an employee perspective to understand how it affects gradually the health and well being apart form also affecting organization loyalty and commitment.

This topic is of great interest as today organization is spending a lot on employee productivity, performance and maintenance of talent, but if issues like presenteeism prevail it may create difficulty for the whole organization. Thus it can be said as one way of sickness but still employees are present. Health and well- being are amongst the key agenda for country's growth and perspective that is one reason why apart from absenteeism in which employees do not report and presenteeism in which employees report to workplace even when they are ill. Thus, organization are nowadays focusing more on introducing absence policies and return to work interviews visit to home and focusing on absence score and trigger points which slowly gradually leads to health related issues for individuals. This can be defined as under pressure to be at work.

\section{Review of Literature}

Sendenet.al(2016) in their study it was found that Female physicians reported sickness presenteeism more often than male physicians. Itis also found in different studies that based on gender the reason of presenteeism also gets affected. In a study conducted by Chatterji and Tilley (2002) also found that different statistical methods can be applied and based on various researches and internal surveysreason affecting presenteeism can be identified.

Vézina et al (2011), in their study found that it is not only the formal methods by which it can be controlled but also by different informal methods which may include looking after their personal needs and other satisfaction causing factors. In a study conducted by Aronsson and Gustafsson (2005) it was found that organization needs to clarify different reactions when it comes to people management, each such action should be based on employee interest well being and outcome ensuring their personal and professional excellence.

Gosselin and Lauzier (2010), however, argue that while efforts to quantify the incidence and costs of presenteeism are still risky, evaluations to date justify continued in.The outcome of presenteeism may lead to consistent loss, lack of productivity and brand name as well A study by Gilbreath and Karimi (2012) investigates 'jobstress-related presenteeism' (aform of psychological strain caused by job stress) and specifically the impact of supervisor behaviour and role modelling. They found that 
job stress and presenteeism were positively correlated and that negative supervisor behaviours had the strongest associations with employee job-stress-related presenteeism.

Lu et al (2013) found that supervisor support acted as a bufferbetween presenteeism and exhaustion. The Health Enhancement ResearchOrganization found that employees who reported it was difficult to eat healthily atwork were 93 per cent more likely to have high presenteeism. Those who reportedthat their employer had little interest in supporting employee efforts to becomingmore physically active were 123 per cent more likely to have high presenteeism.

Problem Statement - Is Presenteeism a more comprehensive productivity paradox than absenteeism?

\section{Research Questions}

- What is the impact and outcome of presenteeism on organization?

- What are the causes of presenteeism on organization and individual perspective?

- What are the different measures to report presenteeism and interventions adopted by organization to reduce its impact?

Research Design - Descriptive type of research.

\section{Objectives of Study}

- To understand the conceptual role of Presenteeism in the Literature.

- To understand the organization and individual reason leading to presenteeism

- To study different methods to measure Presenteeism and interventions to regulate presenteeism

\subsection{Objective 1}

- To understand the conceptual role of Presenteeism in the Literature.

The first objective is explained based on the conceptual literature. From this it is found that there are two key streams of interest in the literature to date (Johns, 2010): Based on reason related to occupational health and safety factor related to culture and other business conditions may affect the level of presenteeism. As stated in the study conducted by Aronsson identified different reasons of why presenteeim prevails which can be due to various factors affecting mental, physical or spiritual health of people but still people keep coming to organization, this may affect organization performance and employee on capability. There can also other reasons related to outside the organization, because of external pressure, industry reason, and country related reasons.

\section{How are Absenteeism and Presenteeism related?}

There is a deep relationship between them as absenteeism matters because it may cause difficulty in various aspects and similarly presenteeism is more harmful as it causes double affect in terms of productivity and performance. There are various researches available about absenteeism but the most important ones include aspects like Periodically occurring issues, it involves active participation of employees in decision making and in suggesting so that ways to avoid causes of presenteeism. Strong reasons, if timely action is not taken then few causes which may appear casual can cause deep effect and loss.

\section{Extreme reason}

These whether related to employee reason of health or professional causes may lead to total loss of talent.

\section{- The difference between mental and physical illness}

The distinction between mental and physical illness is also important because employees believe that it is perceived very differently in the workplace, which could lead to different reporting behaviour. It had been reported that majority people agree that they are not able to report their problem to boss, least people felt that organization takes steps to intervene and resolve problem, it is true that that managers are equipped with the tools they need to be able to confidently and effectively support their staff, whether they are experiencing stress or any other related issues.

\section{- Based on the nature of work}

Based on the nature of work it is thought that Managers should consider and discuss with the employee the mutual impact of work and illness and what circumstances might warrant situation. A checklist because of above three should be prepared and reviewed periodically so that based on these dimensions a consistent review of same can be assessed.

\subsection{Objective 2}

- To understand the organization and individual reason leading to presenteeism

There are several factors that lead to presenteeism right from organizational level, team level or professional

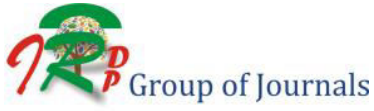


level. Some common reason which the theory reiterates includes, at Professional Level

- Need of others (Affiliation and acceptance needs) - The biggest reason of presenteeism can be because of lack of affiliation or acceptance may be on the part of family, known ones or other acquaintance whether at personal front or at workplace leading to reporting at work place thus causing work place conflcit conditions as well.

- High Concerns for peers: Employees want to spare their time with whenever it comes to higher, additional workload when there is no replacement and will therefore come to work when ill.

- Behavior of Manager and supervisors: It has also been found in several studies that importance of managers and supervisors as role-models. An exemplary behavior on the role of managers needs to exemplify by their behavior, responsiveness and performance.

- Influence of work culture and work ethics and resulting Organization Commitment-Dew et al (2005) describe three very different work cultures which nevertheless all foster presenteeism identified a perception that taking time for development. This work ethic appeared to be passed down as perceived wisdom from older and longer serving employees. These all together lead to organization commitment in way of affective, normative and continuance.

- Organization Policies, Culture and Economic Climate Most of the time irrespective of employee wellness and recognition program etc. it is important that organization policies should be employee centric promoting health, wellness and recognition of employees and ensuring that organization is growth promoting.

- Work time arrangements and sickness benefitsDifferent researchers have found the fact changes in the amount of sickness benefit and how it is paid can have a significant impact on our performance.

One other noteworthy observation is that during the financial crisis, several countries reduced benefits, and this is seen as a key factor driving the declining absenteeism rate (and therefore potential rise in presenteeism) in the various countries. It was seen in different studies that various reasons affect presenteeism like-

- Expectation from Job

- Unclarity in Job or work conditions

- High level of Job stress

- Less rewards

- Low future or benefits to take care of dependents as well

\subsection{Factors related to Work Conditions}

- Exposure to work intensity
- Verbal abuse or discrimination

- Handling chemicals

- Awkward postures

- Shift work.

Personal factors - At the same time as experiencing organisational pressures, individuals have their own motivations for attending work while ill. Some of these relate to circumstances at home that might be unconducive to restor not wanting to suffer alone so that people prefer to be with colleagues.

Vulnerable Groups - There are certain groups as identified in the literature which demonstrate the vulnerable group that are prone to presenteeism with many reasons,

- Insomniacs: This group has a higher absence rate but pose a risk at work as they have a higher accident rate while driving and a three-fold greater risk of having two or three serious road accidents. They also reported poor self-esteem, less efficiency and less job satisfaction at work when, compared with performing sleepers (Léger et al, 2006).

- High-skilled white collar workers: Presenteeism was higher in high-skilled white collar workers (approximately $50 \%$ ), compared to other occupational classes ( 35 to $38 \%$ ), a pattern that observed for mean days of presenteeism (Eurofound, 2012).

- Being childless: Employees who had recently married or those with one child (young child) were less likely to exhibit presenteeism, where as childless employees were more likely to come for work when they were sick.

- Older workers: the image for older workers isn't entirely clear. a number of the conditions like depression, migraine and allergies which are related to presenteeism decrease with age. Others like hypertension, heart condition and back pain increase, et al. remain flat.

- People with unhealthy lifestyle choices: A white book by the Health Enhancement Research Organisation (HERO, 2013) identifies increased risks for smokers, employees with an unhealthy diet and employees who didn't exercise considerably.

- People with poor psychological wellbeing: Presenteeism is significantly related to self-rated anxiety and psychological wellbeing.

- Specific health problems: Various studies identify the risks associated with specific medical conditions. The presence of risk factors, chronic disease, especially chronic depression, radically increases the odds of having high presenteeism.

\subsection{Objective 3}

- To study different methods to measure Presenteeism 


\section{and interventions to regulate absenteeism}

Cost of Presenteeism -people might like better to be with their colleagues than suffer solitude and tedium reception, particularly if they're feeling depressed. On the opposite hand, as presentees struggle on at a decreasing pace, until sometimes finally submitting to an aggravated illness, people who take an entire rest might return to figure quickly at full strength and within the meantime also remove any potential risk to colleagues from infection.

The important components to be considered for presenteesim includes,

- time not on task

- quality of labor (eg mistakes, peak performance, injury rates)

- quantity of labor and private factors (eg social, mental, physical, emotional)

- .Supervisor support: Lu et al (2013) found that supervisor support acted as a buffer between presenteeism and exhaustion.

\section{Challenges in Measuring Presenteeism}

- Self-reported data: Most measures rely solely on the respondents' own perception ofhealth and their evaluation of the legitimacy of taking leave during a given instance thus making it more biased.

- Retrospective frequency measures - These believe recall like looking back over the previous year or shorter periods of your time thus limiting the challenges behind.

- Variety of measurement instruments - there are many sorts of instruments available to live presenteeism but reflect a really limited perspective of reasons of same thus not making it customized.

- Measurement differences across countries - When national pharmacoeconomic guidelines are compared, different recommendations are identified on the way to identify, measure and value lost productivity, resulting in difficulties when comparing lostproductivity estimates across countries.

\section{Available Aspects and Criterion to Live Presenteeism}

- Estimation of Unproductive time while at work - Work Productivity Short Inventory asks employees to estimate what percentage unproductive hours they spent at work during the recall period. Although this approach would cause the easiestmonetisation, Mattke et al (2007) found no examples to show that the employees can exactly transform their perceived impairments into a temporal measure.
- Comparing Productivity, performance and efficiency It helps in benchmarking ones perceived performance thus measuring the identification of possible deviation in performance. This will be easily measured with the supervisor's performance or assessment criteria.

- Assessment instrument supported feedback - With the assistance of self developed reliable and valid inventories it are often identified what proportion and thanks to what causes is that the performance at work thanks to health or illness issues getting affected.

- Estimating the presenteeism from the absenteeism data - Though it's easy to capture data of absenteeism but the info of presenteeism is difficult to assess, however Huver et al (2012) have tested a quantitative tool designed to live presenteeism based on employee absence data.

Using a zero-inflated negative binomial model (ZIMB) and longitudinal data covering 30 years with demographic variables, job-related variables and absence data for every employee from 3,600 employees during a French hospital, they claim to have captured the phenomenon of presenteeism.

The other available tools to live presenteeism includes,

- Stanford Presenteeism Scale (SPS-6): Health Status and Employee Productivity

- World Health Organization Health and Work Performance Questionnaire (HPQ)

The scale by Gilbreath and Frew (2008) (inGilbreath and Karimi, 2012) focuses on work-related stress with six items

\section{Finding of the Study}

- Better training and knowledge for people like managers who work outside the psychological state sphere.

- It is vital that management should ensure giving suitable training/ orientation/ exposure to re orient our people to avoid any quite talent loss.

- Identify those employees returning to figure who are in danger of depression as a key method to figure on your talented employees.

- It is vital for managers at different levels to make sure that they will work closely with each and make suitable interventions to practice techniques to complement culture and make employees more competent.

- To ensure a culture of openness transparency and support centric environment to make sure they're not suffering unnecessary stress.

- Managers should understand the way to apply absence policies effectively and the cultural messages they're intended to offer in order that it's clear that it's a well 
being support mechanism instead of organisational policing.

- Health related programs at work place- it's important to notice that organization got to pay suitable attention to health related issues in organization to avoid absenteeism

- Other interventions include - Conducting effectively planned work health programs like - Involving employees' supervisors / managers in WHP programmes.

- Targeting organisational and/or environmental factors to influence behaviour.

- Screening workers before intervention using HRA (Health Risk Assessment) 3 or other methods.

- Improving supervisor/manager knowledge regarding psychological state within the workplace

\section{Conclusion}

It thus becomes important that presenteeism plays a pivotal role in not only managing presenteeism but also absenteeism and worked upon but when it involves presenteeism the explanations are both visible and invisible thus requires a highly customized and specific approach of counseling, policy wise and a holistic approach towards work excellence and employee engagement.

Presenteesim when looked with a composite perspective can help in improving organization excellence; promote loyalty, talent building and workplace betterment. it'll help in improving work culture and better organization leadership thus improvising working and efficiency. It requires a cooperative and consensus approach on the part of all stakeholders. Thus, it's important to know the role of understanding presenteeism as how of making workforce more productive and culture more enriched.

\section{References}

[1] Ashby and Mahdon, (2010) Why Do Employees Come to Work When Ill? An Investigation into Sickness Presence in the Workplace, The Work Foundation

[2] Aronsson G, Gustafsson K (2005) Sickness presenteeism: prevalence, attendance pressure factors, and an outline of a model for research', Journal of Occupational and Environmental Medicine, Vol. 47, No. 9

[3] Chatterji and Tilley (2002) 'Sickness, absenteeism and sick pay', Oxford Economic Papers, Vol. 54, pp. 669-687

[4] Collins et al, 2005, 'The assessment of chronic health conditions on work performance, absence and total economic impact for employers', Journal of Occupational Environmental Medicine, Vol. 47, pp. 547-57.

[5] Gosselin and Lauzier (2010), Health,absence, disability, and presenteeism cost estimates of certain physical and mental health conditions affecting US employers', Journal of Occupational Environmental Medicine, Vol. 46, pp. 398-412.

[6] Gilbreath and Karimi (2012) 'Supervisor behaviour and employee presenteeism', International Journal of Leadership Studies, Vol. 7, No. 1.

[7] HERO,(2013). New Findings and Realistic Solutions to Employee Presenteeism: A whitepaper from the Health Enhancement Research Organization', Health Enhancement Research Organization [Online]. Available from: http://herohealth.org/researchstudies/

[8] Lu et al (2013) cross-cultural examination of presenteeism and supervisory support', Career Development International, Vol. 18, No. 5

[9] Léger et al,(2006) Professional correlates of insomnia', SLEEP, Vol. 29, No. 2

[10] Parry and Jinnett, (2006), On the Brink of Change: How CFOs View Investments in Health and Productivity, Integrated Benefits Institute.

[11] Parry and Molmen, (2002)The Business Value of Health: Linking CFOs to Health and Productivity, Integrated Benefits Institute.

[12] Ramsey (2006) Presenteeism a new problem in the workplace', Supervision, Vol. 67, No.8.

[13] Senden et.al(2016) Lost productive work time costsfrom health conditions in the United States: results from the American Productivity Audit', Journal of Occupational and Environmental Medicine, Vol. 45, pp. 1234-1246.

[14] Vézina et al (2011) Quebec Survey on Working and Employment Conditions and Occupational Health and Safety, Institute de recherche Robert-Sauvé ensanté et sécurité du travail (IRSST) 